\title{
Sign of tunnel spin polarization of low-work-function Gd/Co nanolayers in a magnetic tunnel junction
}

\author{
B. C. Min, J. C. Lodder, and R. Jansen \\ $\mathrm{MESA}^{+}$Institute for Nanotechnology, University of Twente, 7500 AE Enschede, The Netherlands \\ (Received 3 October 2008; revised manuscript received 7 November 2008; published 4 December 2008)

\begin{abstract}
Magnetic tunnel junctions having a low-work-function $\mathrm{Gd} / \mathrm{Co}$ nanolayer at the interface with an $\mathrm{Al}_{2} \mathrm{O}_{3}$ tunnel barrier are shown to exhibit both positive and negative values of the tunnel magnetoresistance. The sign of the tunnel spin polarization of the $\mathrm{Gd} / \mathrm{Co}$ nanolayer electrode depends on the thickness of the $\mathrm{Gd}$ and Co layers, temperature, and applied voltage. This reflects the nature of the interaction between the conduction electrons of the rare-earth and transition metals.
\end{abstract}

DOI: $10.1103 /$ PhysRevB.78.212403

PACS number(s): 75.70.-i, 72.25.Dc, 73.40.Gk, 85.75.-d

Magnetic tunnel junctions (MTJs), consisting of two ferromagnetic electrodes separated by a thin tunnel barrier, ${ }^{1-3}$ are key elements in spintronic devices such as read heads of magnetic disk drives, magnetic random-access memories, microwave oscillators, and semiconductor-based spin devices. For these applications, engineering the properties of the tunnel contact using new materials is of prime importance. Many opportunities exist, since several factors determine the magnetic response of a MTJ, the tunnel magnetoresistance (TMR), i.e., the relative change in the tunnel resistance in an applied magnetic field. Of importance are the materials used for electrodes as well as the barrier, the structure of the materials (i.e., crystalline versus amorphous), and the specific electronic, structural, and chemical properties of the interfaces. ${ }^{4}$ Notable examples that demonstrate this are the opposite sign of the tunnel spin polarization (TSP) of Co on $\mathrm{Al}_{2} \mathrm{O}_{3}$ and $\mathrm{SrTiO}_{3}$ barriers, ${ }^{5}$ and the enhanced tunnel spin polarization for junctions with epitaxial $\mathrm{MgO}$ barriers showing strong spin filtering due to the symmetry of the wave functions. ${ }^{2,3,6}$ Moreover, even for a fixed combination of materials the TSP can change sign as recently shown for $\mathrm{SrTiO}_{3} / \mathrm{Co}$ interfaces, ${ }^{7,8}$ for example, depending on the terminating layer of the barrier. ${ }^{8}$

For semiconductor-based spin devices such as spin transistors with ferromagnetic source and drain contacts, it was recently emphasized that in addition to the TSP, also the energy band profile of the semiconductor near the tunnel contact should be controlled in order to achieve significant spin signals. ${ }^{9-11}$ In particular, the work function of the ferromagnetic electrode is a crucial parameter that allows for the necessary suppression of the Schottky barrier and the depletion region in the semiconductor. ${ }^{10}$ For spin-tunnel contacts to $\mathrm{Si}$, we have shown that tuning of the Schottky barrier height and the resistance area product of the contacts over a wide range can be achieved by inserting a nanolayer of a low-work-function material such as Gd at the interface between the tunnel barrier and ferromagnetic (FM) electrode. ${ }^{10}$

With respect to the TSP it is noteworthy that previous work by Meservey et al. ${ }^{12}$ on heavy rare-earth metals (Gd, $\mathrm{Tb}$, Dy, Ho, Er, and Tm) on an $\mathrm{Al}_{2} \mathrm{O}_{3}$ tunnel barrier showed that the TSP does not scale with the total magnetic moment. Instead, the TSP was found to be approximately proportional to the magnetic moment of the $5 d$ and $6 s$ conduction electrons. For $\mathrm{Gd}$, the localized $4 f$ electrons provide the main contribution of $+7 \mu_{B}$ to the magnetic moment, ${ }^{12,13}$ while the $5 d 6 s^{2}$ conduction electrons contribute only $+0.63 \mu_{B}$ (with $\mu_{B}$ the Bohr magneton). However, tunneling from $\mathrm{Gd}$ is dominated by states associated with the $5 d$ and $6 s$ conduction electrons, while the $4 f$ electrons of Gd do not contribute to the TSP due to their much more localized nature, causing the wave function to decay rapidly in the tunnel barrier. The measured TSP of $\mathrm{Al}_{2} \mathrm{O}_{3} / \mathrm{Gd}$ is $13 \%$ and positive, ${ }^{12}$ corresponding to predominant tunneling of majority spin electrons. The sensitivity of tunneling to the conduction electrons offers an opportunity to determine the sign of the intraatomic exchange between the $4 f$ and the conduction electrons of rare-earth metals, as well as the interatomic exchange between rare-earth and transition-metal elements, which is otherwise not straightforward to obtain. ${ }^{13}$ For GdCo alloys it was recently shown ${ }^{14}$ that the sign and magnitude of the TMR can be controlled by the alloy composition and temperature as a result of the antiferromagnetic interaction between the transition-metal and rare-earth elements. The spin polarization of the conduction electrons in rare-earth ferromagnets that are believed to mediate the antiferromagnetic interaction was also probed via the giant magnetoresistance effect. ${ }^{15}$ Due to the antiferromagnetic coupling, a sign inversion of the TSP is to be expected when a nanolayer of $\mathrm{Gd}$ is inserted between the tunnel barrier and the transitionmetal electrode. However, in previous work we did not find this sign inversion for the TSP of the $\mathrm{Al}_{2} \mathrm{O}_{3} / \mathrm{Gd} / \mathrm{Ni}_{80} \mathrm{Fe}_{20}$ contacts, ${ }^{10}$ the TSP remaining positive for $\mathrm{Gd}$ nanolayers of up to $2 \mathrm{~nm}$ thick. Here we report on the tunnel spin polarization of $\mathrm{Al}_{2} \mathrm{O}_{3} /$ nanolayer/ $\mathrm{Ni}_{80} \mathrm{Fe}_{20}$ interfaces where the nanolayer is a bilayer of ultrathin Gd and Co layers with total thickness below $1.2 \mathrm{~nm}$. We find that the TSP of such electrodes can be positive or negative depending on the relative thickness of the Gd and Co layers, temperature, and applied voltage.

To determine the TSP of the $\mathrm{Al}_{2} \mathrm{O}_{3} / \mathrm{Gd} / \mathrm{Co} / \mathrm{Ni}_{80} \mathrm{Fe}_{20}$ electrode, MTJs with a counter electrode $(\mathrm{Co})$ of well-known TSP are fabricated. The TMR is measured as TMR $=\left(R_{\mathrm{AP}}\right.$ $\left.-R_{\mathrm{P}}\right) / R_{\mathrm{P}}$, where $R_{\mathrm{P}}\left(R_{\mathrm{AP}}\right)$ is the tunnel resistance for parallel (antiparallel) alignment of the magnetization of the two electrodes. The measured TMR can be expressed as TMR $=2 P_{1} P_{2} /\left(1-P_{1} P_{2}\right)$, where $P_{1}$ and $P_{2}$ are the TSP of the FM/barrier electrodes. Hence, if the TSP of the bottom Co electrode is known, ${ }^{16}$ the TSP of the other 
TABLE I. Layer thickness and TMR of MTJs consisting of $\mathrm{Co}(8 \mathrm{~nm}) / \mathrm{Al}_{2} \mathrm{O}_{3}(3 \mathrm{~nm}) / \mathrm{Gd}\left(t_{\mathrm{Gd}} \mathrm{nm}\right) / \mathrm{Co}\left(t_{\mathrm{Co}} \mathrm{nm}\right) /$ $\mathrm{Ni}_{80} \mathrm{Fe}_{20}(15 \mathrm{~nm}) / \mathrm{CoO}(2.5 \mathrm{~nm}) / \mathrm{Al}(1 \mathrm{~nm})$ at different temperature. The $t_{\mathrm{Gd}}\left(t_{\mathrm{Co}}\right)$ is the thickness of the $\mathrm{Gd}(\mathrm{Co})$.

\begin{tabular}{lccccc}
\hline \hline $\begin{array}{l}\text { Sample } \\
\text { no. }\end{array}$ & $\begin{array}{c}t_{\mathrm{Gd}} \\
(\mathrm{nm})\end{array}$ & $\begin{array}{c}t_{\mathrm{Co}} \\
(\mathrm{nm})\end{array}$ & $\begin{array}{c}\text { TMR } \\
(10 \mathrm{~K}) \\
(\%)\end{array}$ & $\begin{array}{c}\text { TMR } \\
(100 \mathrm{~K}) \\
(\%)\end{array}$ & $\begin{array}{c}\text { TMR } \\
(200 \mathrm{~K}) \\
(\%)\end{array}$ \\
\hline 1 & 0.0 & 1.0 & 28.5 & 26.7 & 20.6 \\
2 & 0.2 & 1.0 & 23.3 & 19.8 & 11.8 \\
3 & 0.4 & 0.6 & 23.4 & 17.5 & 9.9 \\
4 & 0.6 & 0.6 & 19.9 & 13.2 & 6.3 \\
5 & 0.8 & 0.2 & 7.3 & 3.3 & 2.1 \\
6 & 1.0 & 0.2 & -3.7 & -0.8 & 0.6 \\
\hline \hline
\end{tabular}

$\mathrm{Al}_{2} \mathrm{O}_{3} / \mathrm{Gd} / \mathrm{Co} / \mathrm{Ni}_{80} \mathrm{Fe}_{20}$ electrode can be inferred. The full MTJ stack, fabricated on $\mathrm{Al}_{2} \mathrm{O}_{3}$ (0001) substrates, consists of $\mathrm{Co}(8 \mathrm{~nm}) / \mathrm{Al}_{2} \mathrm{O}_{3}(3 \mathrm{~nm}) / \mathrm{Gd}\left(t_{\mathrm{Gd}} \mathrm{nm}\right) / \mathrm{Co}\left(t_{\mathrm{Co}} \mathrm{nm}\right) /$

$\mathrm{Ni}_{80} \mathrm{Fe}_{20}(15 \mathrm{~nm}) / \mathrm{CoO}(2.5 \mathrm{~nm}) / \mathrm{Al}(1 \mathrm{~nm})$. The Co and $\mathrm{Gd}$ layer thicknesses $t_{\mathrm{Gd}}$ and $t_{\mathrm{Co}}$ are summarized in Table I. To pin the magnetization of the top electrode, the $\mathrm{Ni}_{80} \mathrm{Fe}_{20}$ is exchange biased by a $\mathrm{CoO}$ layer formed by Co deposition $(2.5 \mathrm{~nm})$ and in situ plasma oxidation. The blocking temperature of $\mathrm{CoO}(\sim 240 \mathrm{~K})$ limits the temperature range of the exchange bias and thus the measurement. MTJs in fourterminal cross-geometry were prepared using shadow masks as previously described. ${ }^{10}$

Figure 1 shows the TMR versus the applied magnetic field for MTJs with various $\mathrm{Gd} / \mathrm{Co}$ nanolayers measured at $10 \mathrm{mV}$ at $10 \mathrm{~K}$. For each curve, the tunnel resistance changes when the Co bottom electrode magnetization reverses at fields of \pm 50 Oe, while the top electrode magnetization remains fixed in the field range displayed due to the exchange bias. The MTJs with thinner Gd layer $\left(t_{\mathrm{Gd}}<0.6 \mathrm{~nm}\right)$ have a reasonably high value of TMR, but the MTJ with $0.8 \mathrm{~nm} \mathrm{Gd}$ has much smaller TMR (7\%). The most striking result is that the MTJ with $1.0 \mathrm{~nm} \mathrm{Gd} / 0.2 \mathrm{~nm}$ Co shows a clearly negative TMR. For comparison, a positive TMR of $18 \%$ was previously reported ${ }^{10}$ for a $\mathrm{Gd}$ nanolayer of the same $1 \mathrm{~nm}$ thickness, but in direct contact with the $\mathrm{Ni}_{80} \mathrm{Fe}_{20}$ electrode (i.e., without the $0.2 \mathrm{~nm} \mathrm{Co}$ ). This shows that the sign of the TSP is extremely sensitive to the type of transition-metal element.

Figure 2 shows the TSP of the Gd/Co nanolayer top electrode as a function of the Gd thickness at a temperature of 10,100 , and $200 \mathrm{~K}$, calculated from the TMR using known values of the TSP of the Co bottom electrode. ${ }^{10,16}$ At $10 \mathrm{~K}$, the TSP of the nanolayer decreases slightly with increasing Gd thickness up to $0.6 \mathrm{~nm}$, showing a positive value higher than $25 \%$. As the Gd thickness increases further, the TSP decreases steeply, crosses zero, and becomes negative for the largest $t_{\mathrm{Gd}}$. With increasing temperature, the TSP for small Gd thickness remains positive, but the TSP at $1 \mathrm{~nm}$ of Gd changes sign from negative $(T<100 \mathrm{~K})$ to positive $(T$ $>200 \mathrm{~K})$. The sign reversal as a function of composition and temperature leads to small TSP values near the transition, which is a disadvantage when such nanolayers are to be used to lower the work function in spin-tunnel contacts to semiconductors. ${ }^{10}$ Thus, care has to be taken in choosing the

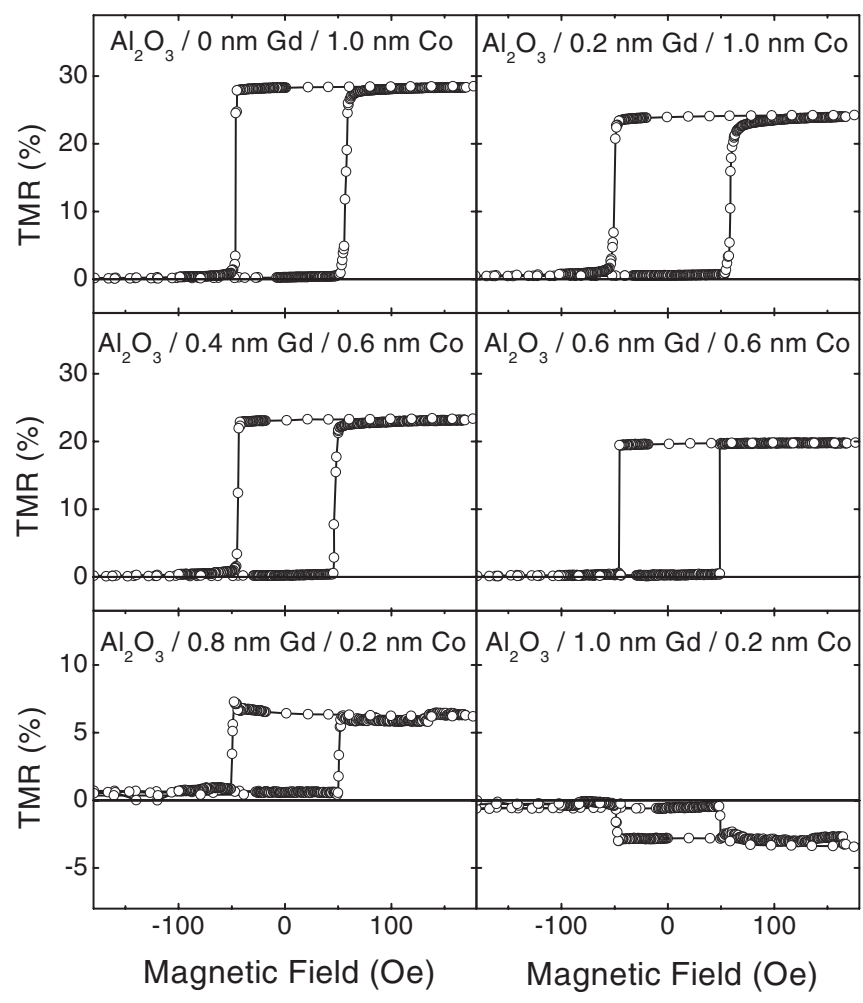

FIG. 1. TMR of MTJs with a Gd/Co nanolayer vs magnetic field measured at $10 \mathrm{mV}$ and $10 \mathrm{~K}$. The MTJs consist of $\mathrm{Co}(8 \mathrm{~nm}) / \mathrm{Al}_{2} \mathrm{O}_{3}(3 \mathrm{~nm}) / \mathrm{Gd}\left(t_{\mathrm{Gd}} \mathrm{nm}\right) / \mathrm{Co}\left(t_{\mathrm{Co}} \mathrm{nm}\right) /$ $\mathrm{Ni}_{80} \mathrm{Fe}_{20}(15 \mathrm{~nm}) / \mathrm{CoO}(2.5 \mathrm{~nm}) / \mathrm{Al}(1 \mathrm{~nm})$. Due to the exchange bias from the $\mathrm{CoO}$, only the bottom Co electrode switches its magnetization direction in the field range displayed. The magnetic moments of the top $\mathrm{Ni}_{80} \mathrm{Fe}_{20}$ and bottom Co electrodes are in the parallel (antiparallel) configuration at negative (positive) field.

proper combination of low-work-function elements and transition metal.

To understand the sign of the TSP we consider that the TSP of $\mathrm{Al}_{2} \mathrm{O}_{3} /$ Co is known to be positive, ${ }^{12}$ and that any $\mathrm{Co}$ atoms in the nanolayer will have their magnetic moment aligned parallel to the magnetization of the main $\mathrm{Ni}_{80} \mathrm{Fe}_{20}$ electrode due to the strong ferromagnetic coupling between transition-metal atoms. ${ }^{13}$ Thus, the junction with only $1 \mathrm{~nm}$

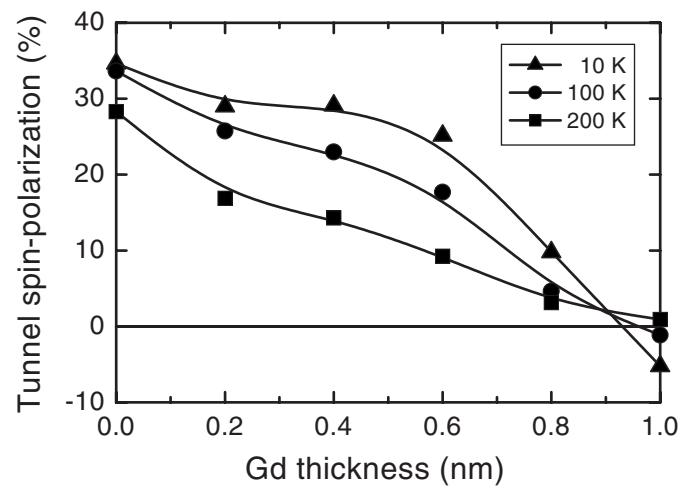

FIG. 2. The TSP of $\mathrm{Al}_{2} \mathrm{O}_{3} / \mathrm{Gd}\left(t_{\mathrm{Gd}} \mathrm{nm}\right) / \mathrm{Co}\left(t_{\mathrm{Co}} \mathrm{nm}\right) /$ $\mathrm{Ni}_{80} \mathrm{Fe}_{20}(15 \mathrm{~nm})$ interfaces as a function of $t_{\mathrm{Gd}}$ measured at 10 , 100 , and $200 \mathrm{~K}$. The solid lines are a guide for the eyes. 
of Co but no Gd has a positive TSP, as expected (see Fig. 2). This implies that the negative TSP observed for thicker Gd layers is due to $\mathrm{Gd}$. It has previously been found ${ }^{12}$ that tunneling from pure $\mathrm{Gd}$ is dominated by states associated with the $5 d$ and $6 s$ conduction electrons, while the $4 f$ electrons of Gd do not contribute to the TSP due to their much more localized nature. A consistent picture then emerges, in which (i) the magnetic moment of the Gd (dominated by the $4 f$ electrons) is aligned antiparallel to the Co and $\mathrm{Ni}_{80} \mathrm{Fe}_{20}$, and (ii) the tunneling electrons associated with the Gd have a spin polarization that is also aligned antiparallel to the Co and the main $\mathrm{Ni}_{80} \mathrm{Fe}_{20}$ top electrode. It follows that the tunneling electrons associated with the Gd have a spin polarization aligned parallel to the Gd moment, consistent with the positive TSP found for pure $\mathrm{Gd}$ electrodes on $\mathrm{Al}_{2} \mathrm{O}_{3}$ (Ref. 12).

Quantum well states in the nanolayer can, in principle, also cause a sign reversal of the TMR. ${ }^{17-19}$ However, conclusive evidence of quantum well oscillations (as opposed to a single sign reversal of the TMR) has only been given for high-quality epitaxial structures, where the roughness can be made smaller than the oscillation period. As also pointed out by LeClair et al. ${ }^{20}$ for Ru nanolayers, quantum well states are rather unlikely in an MTJ with an amorphous tunnel barrier and polycrystalline electrodes, since even the slightest lateral variations in the nanolayer thickness (or in the extent of intermixing) will cause the oscillations to average out. Hence, significant contributions of quantum well oscillations to the TMR are not likely for our structures. Moreover, the negative TMR observed in the uniform CoGd alloys ${ }^{14}$ also suggests that the negative TMR we observe is due to the antiferromagnetic coupling of $\mathrm{Co}$ and $\mathrm{Gd}$, rather than due to quantum well states.

We note that some mixing of the Gd and Co in the nanolayer can be expected. It is known that asymmetric diffusion occurs at a $\mathrm{Co} / \mathrm{Gd}$ interface where Co diffuses into the $\mathrm{Gd}$ layers and forms a $\mathrm{Gd}_{x} \mathrm{Co}_{1-x}$ alloy that is limited at nearly the eutectic composition $\left(\mathrm{Gd}_{63} \mathrm{CO}_{37}\right)$ (Ref. 21). A different $\mathrm{Co} / \mathrm{Gd}$ bilayer configuration may thus result in a different Co-Gd alloy composition at the $\mathrm{Al}_{2} \mathrm{O}_{3}$ surface. While this may affect how the TSP varies for intermediate $t_{\mathrm{Gd}}$, it leaves intact the interpretation of the sign reversal as a transition from positive TSP dominated by the Co, to a negative TSP dominated by tunneling electrons associated with Gd. This interpretation is consistent with previous work on $\mathrm{Gd} / \mathrm{Co}$ alloys, ${ }^{14}$ although it should be noted that the main focus there was on a different phenomenon, namely, the sign reversal of TSP around the compensation point of the alloy, causing all the magnetic moments in the alloy to reverse sign with respect to the applied magnetic field.

In addition to the sign reversal with composition of the $\mathrm{Gd} / \mathrm{Co}$ nanolayer, we also find that the TSP sign depends on bias voltage, an aspect that has not been reported previously. Figure 3 shows the TMR versus bias voltage for the same MTJs. When Gd is introduced into the nanolayer, the TMR decays faster with bias [compare, for instance, (a) and (c)]. However, the bias dependence for thicker Gd in Figs. 3(e) and 3(f) is more peculiar. The TMR in Fig. 3(e) at zero bias is $+5 \%$. With increasing positive or negative bias, the TMR initially increases up to the maximum of $+14 \%$, but then

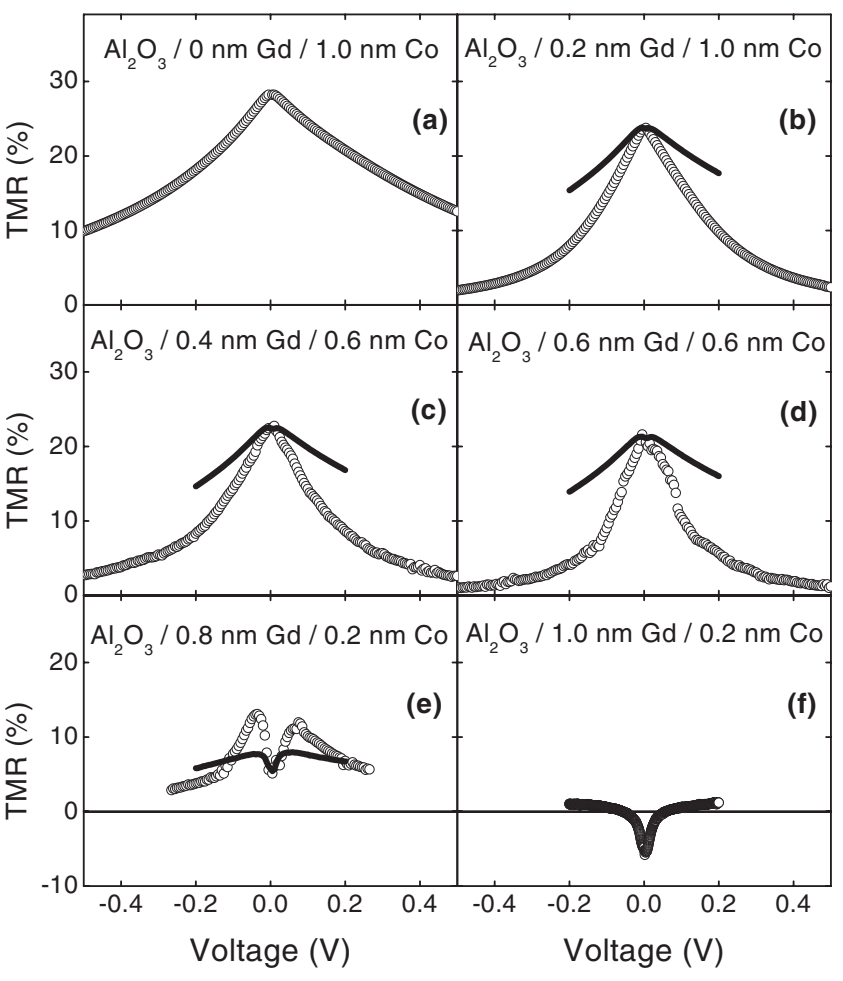

FIG. 3. TMR versus applied bias voltage of MTJs with a Gd/Co interfacial nanolayer. For negative bias, electrons tunnel from the $\mathrm{Gd} / \mathrm{Co}$ nanolayer into the Co bottom electrode. Symbols are the experimental data; the solid lines in (b)-(e) are obtained from a linear combination of spin-polarized tunneling from $\mathrm{Co}$ and $\mathrm{Gd}$ using the tunnel characteristics of (a) and (f), as explained in the text.

starts to decrease. The TMR in Fig. 3(f) at zero bias starts at a negative value of $-5 \%$. With increasing bias the TMR crosses zero and becomes positive. We thus find that the negative TSP is most pronounced at low bias, where states close to the Fermi level dominate the tunneling process.

In previous work ${ }^{14,22}$ on tunnel junctions with homogeneous alloy electrodes $(\mathrm{Gd} / \mathrm{Co}$ or $\mathrm{Pt} / \mathrm{Co})$, the sign and magnitude of the TSP were interpreted as a weighted sum of independent tunneling from the different atomic species, each with their own tunneling probability and tunneling spin polarization. We have tested this picture against the data in Fig. 3. We take a weighted linear combination of the current versus bias voltage for the case of Co [represented by the junction in (a)] and Gd [represented by the junction in (f)], for parallel electrode magnetization. Then we do the same for the antiparallel situation, and from that compute the TMR versus voltage for each of the intermediate curves (b)-(e). Thus, we use $I^{\mathrm{P}, \mathrm{AP}}=\alpha I^{\mathrm{P}, \mathrm{AP}}(\mathrm{Co})+\beta I^{\mathrm{P}, \mathrm{AP}}(\mathrm{Gd})$, where $\alpha$ and $\beta$ are the weight factors for the $\mathrm{Co}$ and $\mathrm{Gd}$ contribution to the tunneling current, respectively. For the solid curves in Figs. 3(b)-3(e) that represent the expected TMR versus bias voltage in this model, we used the weight factors $(\alpha, \beta)$ $=(0.9,0.1),(0.87,0.13),(0.84,0.16)$, and $(0.4,0.6)$, respectively. The weight factors are adjusted such that the resulting TMR value at zero bias matches with the experimental data. It is clear that there are large discrepancies with the actual data. Thus, the simple picture, in which the tunnel spin po- 
larization of an alloy electrode is represented by a weighted sum of spin-polarized tunneling of the individual atomic species, does not describe the TMR versus bias voltage very well. Hence, the interaction of the rare-earth and the transition-metal elements and the hybridization of their wave functions have to be considered, and the electronic structure of the alloy as a whole has to be taken into account.

The results show that spin-polarized tunneling can be used to probe magnetic interactions in systems containing rare-earth and transition metals. The sign of the TSP of the $\mathrm{Gd} / \mathrm{Co}$ nanolayer electrodes reveals that the tunneling electrons associated with $\mathrm{Gd}$ have a spin polarization aligned ferromagnetically to the Gd $4 f$ moment, but antiparallel to the moment of the Co transition-metal atoms in the nanolayer. The TMR variation with bias voltage shows that the interaction between the rare-earth and transition-metal elements modifies their electronic structure, such that a picture in which the tunnel spin polarization of an alloy electrode is represented by a weighted sum of spin-polarized tunneling of the individual atomic species is not appropriate.

The authors acknowledge financial support from the Netherlands Nanotechnology Networks, NANOIMPULS and NANONED (supported by the Ministry of Economic Affairs).
${ }^{1}$ J. S. Moodera, L. R. Kinder, T. M. Wong, and R. Meservey, Phys. Rev. Lett. 74, 3273 (1995).

${ }^{2}$ S. S. P. Parkin, C. Kaiser, A. Panchula, P. M. Rice, B. Hughes, M. Samant, and S.-H. Yang, Nature Mater. 3, 862 (2004).

${ }^{3}$ S. Yuasa, T. Nagahama, A. Fukushima, Y. Suzuki, and K. Ando, Nature Mater. 3, 868 (2004).

${ }^{4}$ J. P. Velev, P. A. Dowben, E. Y. Tsymbal, S. J. Jenkins, and A. N. Caruso, Surf. Sci. Rep. 63, 400 (2008).

${ }^{5}$ J. M. De Teresa, A. Barthelemy, A. Fert, J. P. Contour, F. Montaigne, and P. Seneor, Science 286, 507 (1999).

${ }^{6}$ W. H. Butler, X.-G. Zhang, T. C. Schulthess, and J. M. MacLaren, Phys. Rev. B 63, 054416 (2001).

${ }^{7}$ A. Thomas, J. S. Moodera, and B. Satpati, J. Appl. Phys. 97, 10C908 (2005).

${ }^{8}$ I. J. Vera Marún, F. M. Postma, J. C. Lodder, and R. Jansen, Phys. Rev. B 76, 064426 (2007).

${ }^{9}$ A. Fert and H. Jaffrès, Phys. Rev. B 64, 184420 (2001).

${ }^{10}$ B. C. Min, K. Motohashi, J. C. Lodder, and R. Jansen, Nature Mater. 5, 817 (2006).

${ }^{11}$ R. Jansen and B. C. Min, Phys. Rev. Lett. 99, 246604 (2007).

${ }^{12}$ R. Meservey and P. M. Tedrow, Phys. Rep. 238, 173 (1994).

${ }^{13}$ Magneto-optical Recording Materials, edited by R. J. Gambino and T. Suzuki (IEEE, New York, 2000); Handbook of Magnetic Materials, edited by K. H. J. Buschow (North-Holland, Amsterdam, 1991), Vol. 6; K. Moorjani and J. M. D. Coey, Magnetic
Glasses (Elsevier, Amsterdam, 1984).

${ }^{14}$ C. Kaiser, A. F. Panchula, and S. S. P. Parkin, Phys. Rev. Lett. 95, 047202 (2005); C. Kaiser and S. S. P. Parkin, Appl. Phys. Lett. 88, 112511 (2006).

${ }^{15}$ A. T. Hindmarch and B. J. Hickey, Phys. Rev. Lett. 91, 116601 (2003).

${ }^{16}$ B. G. Park, T. Banerjee, B. C. Min, J. G. M. Sanderink, J. C. Lodder, and R. Jansen, J. Appl. Phys. 98, 103701 (2005); B. G. Park, T. Banerjee, J. C. Lodder, and R. Jansen, Phys. Rev. Lett. 99, 217206 (2007).

${ }^{17}$ J. S. Moodera, J. Nowak, L. R. Kinder, P. M. Tedrow, R. J. M. van de Veerdonk, B. A. Smits, M. van Kampen, H. J. M. Swagten, and W. J. M. de Jonge, Phys. Rev. Lett. 83, 3029 (1999).

${ }^{18}$ S. Yuasa, T. Nagahama, and Y. Suzuki, Science 297, 234 (2002).

${ }^{19}$ T. Nozaki, Y. Jiang, Y. Kaneko, A. Hirohata, N. Tezuka, S. Sugimoto, and K. Inomata, Phys. Rev. B 70, 172401 (2004).

${ }^{20}$ P. LeClair, B. Hoex, H. Wieldraaijer, J. T. Kohlhepp, H. J. M. Swagten, and W. J. M. de Jonge, Phys. Rev. B 64, 100406(R) (2001).

${ }^{21}$ J. P. Andrés, J. L. Sacedón, J. Colino, and J. M. Riveiro, J. Appl. Phys. 87, 2483 (2000).

${ }^{22}$ C. Kaiser, S. van Dijken, S.-H. Yang, H. Yang, and S. S. P. Parkin, Phys. Rev. Lett. 94, 247203 (2005). 